\title{
Phase Transition and Magnetoelectric Effect in 2D Ferromagnetic Films on a Ferroelectric Substrate
}

\author{
Igor Bychkov $^{1, * \mathbb{D}}$, Sergey Belim ${ }^{2, * \mathbb{D}}$, Ivan Maltsev ${ }^{1}$ and Vladimir Shavrov $^{3} \mathbb{D}$ \\ 1 Faculty of Physics, Chelyabinsk State University, 454001 Chelyabinsk, Russia; malts_iv@mail.ru \\ 2 Faculty of Radiophysics, Omsk State Technical University, 644050 Omsk, Russia \\ 3 Kotelnikov IRE RAS, 125009 Moscow, Russia; shavrov@cplire.ru \\ * Correspondence: bychkov@csu.ru (I.B.); sbelim@mail.ru (S.B.)
}

check for updates

Citation: Bychkov, I.; Belim, S.;

Maltsev, I.; Shavrov, V. Phase

Transition and Magnetoelectric Effect in 2D Ferromagnetic Films on a Ferroelectric Substrate. Coatings 2021,

11, 1325. https://doi.org/ 10.3390/coatings 11111325

Academic Editor: Joe Sakai

Received: 25 September 2021

Accepted: 27 October 2021

Published: 29 October 2021

Publisher's Note: MDPI stays neutral with regard to jurisdictional claims in published maps and institutional affiliations.

Copyright: (c) 2021 by the authors. Licensee MDPI, Basel, Switzerland. This article is an open access article distributed under the terms and conditions of the Creative Commons Attribution (CC BY) license (https:// creativecommons.org/licenses/by/ $4.0 /)$.
Abstract: In this paper, we investigate the behavior of 2D ferromagnetic (FM) films on a ferroelectric (FE) substrate with a periodic structure. The two-dimensional Frenkel-Kontorova (FK) potential simulates the substrate effect on the film. The substrate potential corresponds to a cubic crystal lattice. The Ising model and the Wolf cluster algorithm are used to describe the magnetic behavior of a FM film. The effect of an electric field on a FE substrate leads to its deformation, which is uniform and manifests itself in a period change of the substrate potential. Computer simulation shows that substrate deformations lead to a decrease in the FM film Curie temperature. If the substrate deformations exceed 5\%, the film deformations become inhomogeneous. In addition, we derive the dependence of film magnetization on the external electric field.

Keywords: ferromagnetic; magnetoelastic; phase transition; thin films; Frenkel-Kontorova potential

\section{Introduction}

A wide range of spintronic devices uses the magnetoelectric (ME) effect in two-layer systems. The ME effect allows controlling magnetization with an external electric field. That, in turn, allows managing the conductivity of the system due to the phenomenon of giant magnetoresistance. A giant ME effect occurs in systems consisting of a thin FM film deposited on a FE substrate. One of the ME effect appearance mechanisms in such systems shows up due to the shift in the Curie point in the FM film under the substrate influence. The deformation of the FE substrate occurs in an external electric field. The film is also deformed due to the interaction of the atoms of the film and the substrate. Film deformation due to magnetostrictive phenomena leads to a shift in the Curie temperature. At a constant temperature, the electric field shifts the system position in the phase diagram relative to the phase transition point, which leads to a change in magnetization.

Experiments show that a change in the magnetization of thin films under the substrate influence occurs for various materials. Ultrathin platinum films on a $\mathrm{BaTiO}_{3} \mathrm{FE}$ substrate [1] significantly change the value of their magnetic moment upon substrate deformation on an external electric field. The ME effect was observed in Ni films on the same substrate [2]. The use of diffraction and reflection of X-rays in thin $\mathrm{Pt} / \mathrm{Co} / \mathrm{Ta}$ films on various $\mathrm{FE}$ substrates [3] in an external electric field allows observing the change in the magnetic domains structure. A large magnitude of the ME effect was noted in thin FM films $\mathrm{CoFe}_{2} \mathrm{O}_{3} / \mathrm{Pb}\left(\mathrm{Zr}_{0.52} \mathrm{Ti}_{0.48}\right) \mathrm{O}_{3} / \mathrm{LaNiO}_{3}$ on a $\mathrm{Pt} / \mathrm{Ti} / \mathrm{SiO}_{2} / \mathrm{Si}$ substrate with the coexistence of FE and FM phases [4]. A study of the change in the magnetic moment in a $\mathrm{Ni}_{80} \mathrm{Co}_{20}$ film on a $\mathrm{Pb}(\mathrm{Mg}, \mathrm{Nb}) \mathrm{O}_{3}-\mathrm{PbTiO}_{3}$ substrate under the influence of an external electric field is represented in [5]. This study revealed that the change in magnetization is a consequence of substrate deformations. A large $\mathrm{ME}$ effect of $560 \mathrm{mVcm}^{-1} \mathrm{E}^{-1}$ occurs in the $\mathrm{Pb}\left(\mathrm{Zr}_{0.52} \mathrm{Ti}_{0.48}\right) \mathrm{O}_{3} / \mathrm{LaNiO}_{3} / \mathrm{Ni}$ [6] heterostructure, where $\mathrm{LaNiO}_{3}$ serves as a buffer layer. $\mathrm{Ba}_{0 .} \mathrm{Ca}_{0.1} \mathrm{TiO}_{3} / \mathrm{CoFe}_{2} \mathrm{O}_{4}$ thin films on $\mathrm{Pt} / \mathrm{Ti} / \mathrm{SiO}_{2} / \mathrm{Si}$ [7] substrates exhibit a fairly 
large ME effect $\left(82 \mathrm{mVcm}^{-1} \mathrm{E}^{-1}\right)$. FM films $\mathrm{La}_{0.7} \mathrm{Ca}_{0.3} \mathrm{MnO}_{3}$ on a FE substrate $\mathrm{BaTiO}_{3}$ demonstrate a Curie temperature decrease both under tension and under compression [8].

Theoretical studies of the ME effect are focused mainly on specific substances. The microscopic model for $\mathrm{Al}_{2} \mathrm{O}_{3}$ and $\mathrm{MgO}$ substrates [9] became a basis for the investigation of the substrate impact on the magnetic properties of $\mathrm{BaCoF}_{4}$ films. The phase transition temperature and the magnetization of the thin film increase for a compressible substrate, whereas they decrease for a tensile substrate. To study magnetization fluctuations in twodimensional $\mathrm{RMnO}_{3}$ films ( $\mathrm{R}=\mathrm{Tb}, \mathrm{Lu}$, and $\mathrm{Y}$ ) on a FE substrate, the modified Landau model was used [10].

Thus, in this paper, we study the FM phase transition and ME effect in two-dimensional films on a FE substrate from a common understanding of the crystal lattice symmetry and the interaction of atoms without reference to a specific substance.

\section{Materials and Methods}

We explore a two-dimensional FM film and investigate its magnetic properties within the Ising model. The undeformed film has a square lattice with a period of $a$. The atoms located at the film sites are characterized by the value of the spin $S$, which can take one of two values $(+1 / 2$ or $-1 / 2)$. The Hamiltonian of such a system is as follows:

$$
H=-\sum J\left(r_{i, j}\right) S_{i} S_{j},
$$

where $r_{i j}$ is the distance between atoms $i$ and $j . J\left(r_{i j}\right)$ is the exchange integral. Generally, the exchange interaction between spins decreases exponentially; thus, one can take into account the summation over the nearest neighbors only. When the film is deformed, the distance between the nearest neighbors changes. Therefore, it is necessary to consider the dependence of the exchange integral on the distance as follows:

$$
J\left(r_{i j}\right)=J_{0} e^{\left(-\left|r_{i j}-a\right| / r_{0}\right)},
$$

where $J_{0}$ is the exchange integral in an undeformed FM film. Parameter $r_{0}$ determines the rate of the exchange integral decrease with distance and depends on the specific type of substance. This approximation leads to the fact that the effects of long-range action, which are present in some systems, are not taken into account. Long-range effects require considering the interaction not only with the nearest neighbors, but also with the secondnearest ones, and it can lead to a change in the critical temperature. For short-range systems, the exponential law is not universal as well; exchange interactions can be more complex, but this approximation is basic in spin models. Other forms of exchange integrals can be described using additive corrections to the exponential law; still, this is hard to be covered in a single article.

The influence of substrate manifests itself through the interaction between film atoms and substrate surface atoms. The substrate surface atoms affect geometric position of the film atoms, and this influence depends on their arrangement. In our simulations, we model the substrate as a two-dimensional potential with periodically located potential wells. We restrict ourselves to the case of a substrate with a square lattice. We use the two-dimensional FK potential to describe the film interaction with the surface of such a substrate [11].

$$
U_{s u b}=\frac{A}{2}\left(2-\cos \left(\frac{2 \pi x}{b_{x}}\right)-\cos \left(\frac{2 \pi y}{b_{y}}\right)\right),
$$

where $(x, y)$ are coordinates of a point on the substrate surface, $b_{x}$ and $b_{y}$ are the periods of substrate potential along the OX and OY axis correspondingly, and $A$ is the amplitude of substrate potential. For an undeformed substrate, we assume the square crystal lattice and equal periods along both axes $\left(b_{x}=b_{y}=b\right)$. External electric field leads to the deformation of the substrate crystal along the direction of the strength vector. We assume that deformation depends linearly on the electric field strength $E$ at low values of the 
tension. Within the computer simulation, we apply an external electric field along the OX axis. Thus, the lattice period changes along the OX axis.

$$
\begin{gathered}
b_{x}=\epsilon b, \\
1-\epsilon=\gamma E,
\end{gathered}
$$

where $\epsilon$ is the relative deformation and $\gamma$ is the ferroelectric constant characteristic of the substance.

In addition to interaction with the substrate, mechanical interaction between atoms occurs in a thin film. As in the FK model, we restrict ourselves to the harmonic approximation. The energy of interaction between atoms has the following form:

$$
U_{i n t}=\frac{g}{2} \sum_{n, m}\left(\left(x_{n+1, m}-x_{n, m}-a\right)^{2}+\left(y_{n, m+1}-y_{n, m}-a\right)^{2}\right),
$$

where $g$ is an elastic constant, and $\left(x_{n, m}, y_{n, m}\right)$ are the coordinates of the $(n, m)$ atom.

It is necessary to minimize the total energy of the atomic system to determine the equilibrium position of the film atoms.

$$
U=U_{i n t}+U_{\text {sub }} \rightarrow \min ,
$$

$U=\frac{A}{2}\left(2-\cos \left(\frac{2 \pi x_{n, m}}{b_{x}}\right)-\cos \left(\frac{2 \pi y_{n, m}}{b_{y}}\right)\right)+\frac{g}{2} \sum_{n, m}\left(\left(x_{n+1, m}-x_{n, m}-a\right)^{2}+\left(y_{n, m+1}-y_{n, m}-a\right)^{2}\right)$.

To search for the equilibrium state of atoms in the film, we use the Monte Carlo method. An unperturbed film with atoms at the sites of a square lattice with a period $a$ is considered the initial state. The method of successive iterations determines the equilibrium state. One iteration consists of each lattice atom performing a test shift by a random vector, the length of which does not exceed 0.1a. If the new position reduces the total energy of the system, then it is taken. Otherwise, the atom returns to its previous position. In the equilibrium state, the iteration does not change the atomic arrangement.

The arrangement of film atoms on the substrate is determined by the competition between the interaction of film atoms with each other and with the substrate. For an undeformed system, atoms are located at the minima of the substrate potential. The minima are displaced relative to the initial position with a uniform substrate deformation. As a result, for some atoms, the interatomic interaction dominates over the interaction with the substrate, while for others, the substrate plays a more significant role. This leads to different displacements of atoms from the initial state, which manifests itself in the form of inhomogeneous deformations. Inhomogeneous magnetic film deformations with uniform FE substrate deformations along one axis were experimentally observed using X-ray diffraction in [12].

Having determined the atomic positions in the film equilibrium state, we pass to its magnetic properties. We determine magnetization $m$ as the average spin per node.

$$
m=\sum_{i} S_{i} / N,
$$

where $N$ is the total number of atoms in the film.

We use the finite-size scaling theory [13] to determine the temperature of phase transitions. System evolution proceeds according to the Wolf cluster algorithm [14]. We investigate systems of different linear dimensions $L$. For each of those, the dependence of the fourth-order Binder cumulants on temperature [15] is determined:

$$
U_{4}=1-\frac{\left\langle m^{4}\right\rangle}{3\left\langle m^{2}\right\rangle^{2}} .
$$


Angle brackets denote averaging over thermodynamic states. We find the Curie temperature as the intersection of the graphs of the Binder cumulants versus temperature for systems with different linear dimensions.

\section{Results}

\subsection{Computer Simulation}

We review the systems with linear dimensions from $L=18$ to 204 with the help of computer simulation. To satisfy the periodic boundary condition (if $b \neq a$ ), we must choose different sets of $L$ for each $b$ in such a way that the integer number of the $L$ film atoms has an integer number of potential minima $N_{p}$, i.e., $L a=N_{p} b$, where $L$ and $N_{p}$ integer. The central atom is fixed, and the boundary condition is periodical. The search for the equilibrium state of the film's atoms took 100,000 Monte Carlo steps; the first 100,000 MC steps of the magnetic system evolution are discarded, and the magnetic properties are calculated in the next 50,000 MC steps.

For convenience, we pass to the relative values. We choose the film lattice period as a unit of length $a=1$. We consider systems with undeformed film in the initial state. This state is realized when the periods of the film and substrate coincide $(b=a)$. In this case, the atoms of the film are located at the minima of the substrate potential. An external electric field operates along the OX axis; therefore, substrate deformations also occurred along the OX axis $\left(b_{x}=\epsilon, b=\epsilon\right)$. The period of substrate potential along the OY axis is unchanged $\left(b_{y}=b=1\right)$. We choose the unit value for the FE constant $(|\gamma|=1)$. Different values of the FE constant will lead to changes in the electric field strength, while the main dependencies will not change. We perform calculations in the range of $E$ from 0 to 0.1. The compression or substrate stretching depends on the sign of $\gamma$. Values $\gamma>0$ provide substrate compression and $E=1-\epsilon, \gamma<0$ lead to substrate stretching and $E=\epsilon-1$. For the elastic constant, a unit value $(g=1)$ is also chosen. The units for the amplitude of the substrate potential are the same as that for the elastic constant. We perform the calculations for $A=0.1,0.5$ and 1.0. Figure 1 shows the dependence of Curie temperature on the external electric field strength.

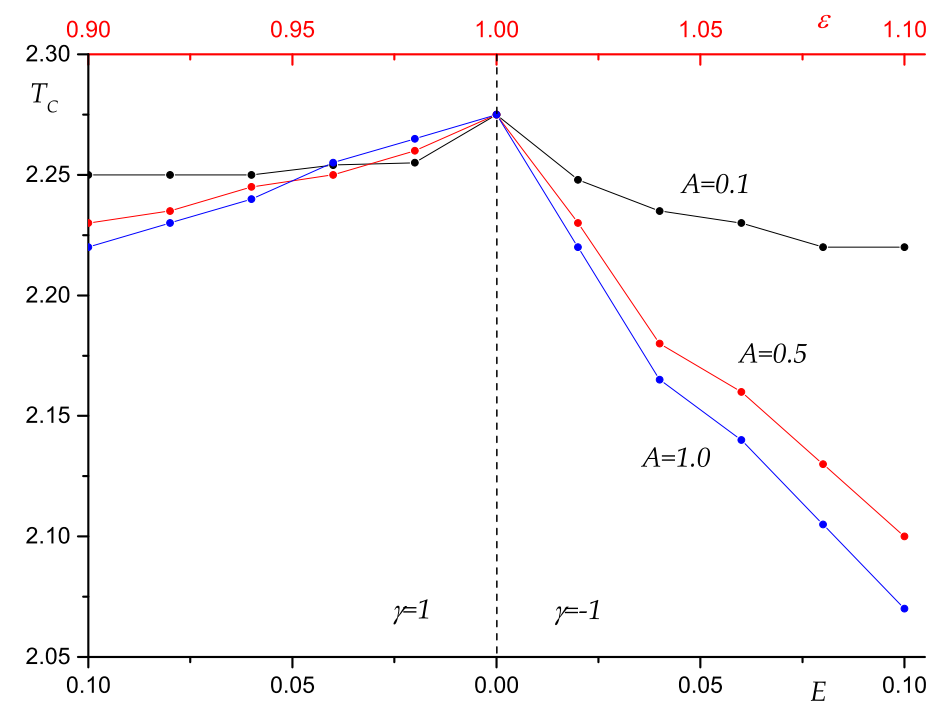

Figure 1. Dependence of the Curie temperature on the strength of the external electric field at different amplitudes of the substrate potential.

It can be seen that substrate deformations under the external electric field lead to a Curie temperature decrease. These results on the Curie temperature behavior are in qualitative agreement with the experimental data [8]. The authors observed a decrease in the phase transition temperature of the epitaxial film $\mathrm{La}_{0.7} \mathrm{Ca}_{0.3} \mathrm{MnO}_{3}$ upon compression 
of the ferroelectric substrate $\mathrm{BaTiO}_{3}$ by $1 \%$ from $240 \mathrm{~K}$ to $160 \mathrm{~K}$. When the substrate was stretched by $6 \%$, the Curie temperature decreased to $195 \mathrm{~K}$. The decrease in the phase transition temperature is associated with rearrangement of the film crystal lattice. Deformations occur unevenly in both cases of compression and tension (Figure 2).

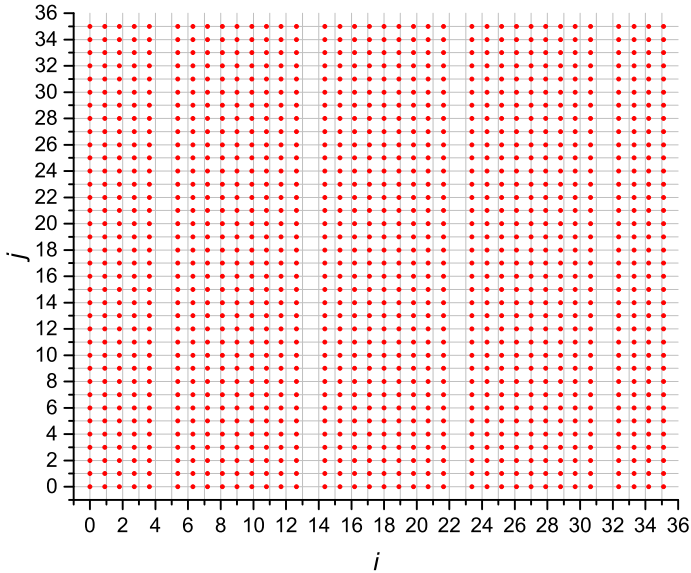

(a)

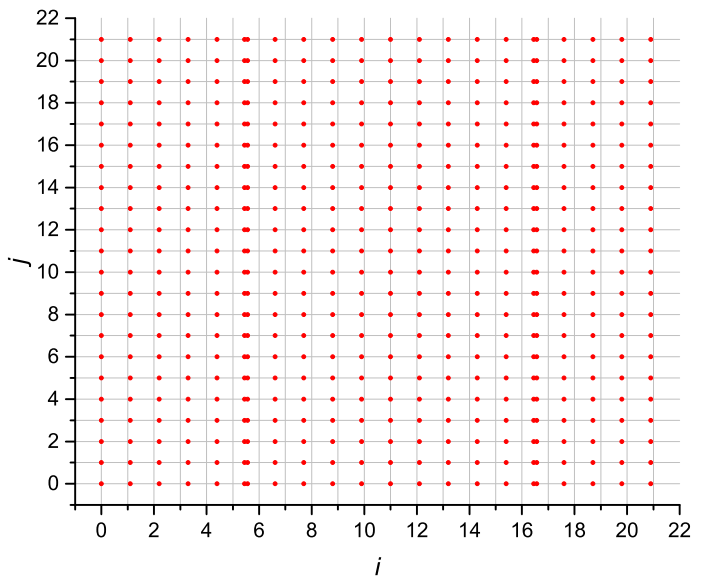

(b)

Figure 2. Atomic arrangement of a FM film during tension (a) and compression (b) of the substrate $(A=1.0)$ (undeforming atomic arrangement falls on the intersection of vertical and horizontal grid lines).

As shown in Figure 2, compression and tension along the OX axis lead to the formation of periodic strip structures in the FM film. In both cases, there is competition between two factors. Upon compression within each stripe, the atomic density increases, which leads to an increase in the Curie temperature. However, the formation of regions of reduced concentration between the stripes leads to a decrease in the phase transition temperature. Stretching inside each stripe decreases the concentration of atoms, leading to a Curie temperature decrease. However, layering of the neighboring stripes leads to an increase in the atoms concentration in the overlap region and an increase in the phase transition temperature. In both cases, the factors lowering the Curie temperature dominate, but the decrease in magnitude is different.

The ME effect consists of a change in the magnetization of the film when the system is in an external electric field. The ME effect is observed in a thin film in the FM phase. Therefore, we consider the system at temperatures below the Curie point. Figure 3 shows the dependence of the thin film magnetization on the strength of the external electric field at three different temperatures.

The magnetization of an FM film decreases in an external electric field, due to the Curie temperature changes. An external electric field brings the system closer to the phase transition point at a fixed temperature, which leads to a decrease in magnetization. 


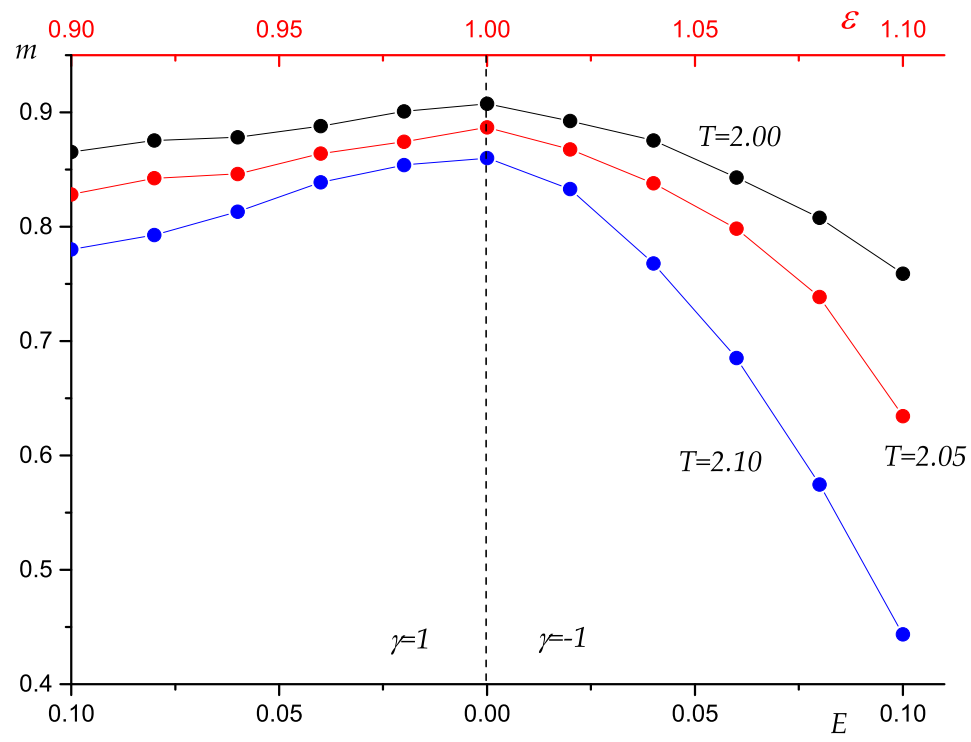

Figure 3. Dependence of the magnetization of a thin film on the strength of the external electric field at three different temperatures for $A=1.0$.

\subsection{Mean-Field Theory}

Let us pass to the model of the system under consideration within the mean-field theory. The free energy of the system can be recorded as follows:

$$
F=F_{M}+F_{D F}+F_{M D}+F_{D S}+F_{S F}+F_{F E} .
$$

Here, $F_{M}$ is the free energy of magnetization of a thin FE film, $F_{D F}$ is the deformation energy of a thin film, $F_{M D}$ is the energy of magnetostriction, $F_{D S}$ is the energy of deformation of a FE substrate, $F_{S F}$ is the energy of the film-substrate interaction, and $F_{F E}$ is the energy of interaction of a FE substrate with an external electric field.

Near the second-order phase transition, the magnetization energy term for an FM film can be written as a series in powers of magnetization [16].

$$
F_{M}=A M^{2}+B M^{4} .
$$

where $B$ is a positive constant.

$$
A=\alpha\left(T-T_{0}\right) .
$$

where $\alpha$ is a positive constant, $T$ is the system temperature, and $T_{0}$ is the intrinsic Curie temperature of the FM film in the absence of external influence.

The free energy with uniform film deformations are as follows:

$$
F_{D F}=C x^{2} .
$$

where $x$ is the change in the linear dimensions of the film, $C$ is the modulus of elasticity of the film.

We can record the energy of elastic deformations of the substrate similarly as follows:

$$
F_{D S}=G y^{2} .
$$

where $y$ is the change in the linear dimensions of the substrate, and $G$ is the elastic modulus of the substrate.

The energy of magnetostriction in most substances is quadratic:

$$
F_{M D}=D M^{2} x .
$$

where $D$ is the positive constant of magnetostriction. 
The interaction energy of a FE substrate with an external electric field $E$ is linear:

$$
F_{F E}=H y E .
$$

where $H$ is a positive constant.

At small deformations of the substrate, we can take into account only the linear interaction between the deformations of the substrate and film.

$$
F_{S F}=R x y
$$

where $R$ is a positive constant.

The free energy of a thin FM film on a FE substrate will have the following form:

$$
F=A M^{2}+B M^{4}+C x^{2}+D M^{2} x+G y^{2}+R x y+H y E .
$$

The condition of the system equilibrium in an external electric field can be expressed as follows:

$$
\left\{\begin{array}{l}
\frac{\partial F}{\partial M}=0 \\
\frac{\partial F}{\partial x}=0 \\
\frac{\partial F}{\partial y}=0 .
\end{array}\right.
$$

Hence, we obtain a system of equations for the equilibrium values of deformations $(x, y)$ and magnetization.

$$
\left\{\begin{array}{l}
2 G y+H E+R x=0 \\
2 C x+D M^{2}+R y=0 \\
2 A M+4 B M^{3}+2 D M x=0 .
\end{array}\right.
$$

Assuming the system is in the FM phase $(M \neq 0)$ and solving this system of equations, we obtain the magnetization dependence of a thin film on the electric field strength applied to the substrate.

$$
M^{2}=\frac{A+D R H E}{D^{2}+2 B R^{2}-8 B C G} .
$$

The general view of magnetization dependence on the electric field strength has the following form:

$$
M^{2}=\alpha+\beta E
$$

Moreover, $\alpha=\alpha_{0}\left(T-T_{0}\right)$.

To compare this conclusion with the results of a computer simulation, we plot the dependence of $M^{2}$ on $E$ at different temperatures (Figure 4).

The behavior of $M^{2}(E)$ curves is close to linear at low electric fields. At $E>0.06$, the dependence ceases to be linear, and the mean-field theory is not applicable. An increase in temperature leads to a parallel displacement of the curves along the OY. The behavior of obtained curves is in qualitative agreement with the conclusions obtained with our model based on the mean-field theory. 


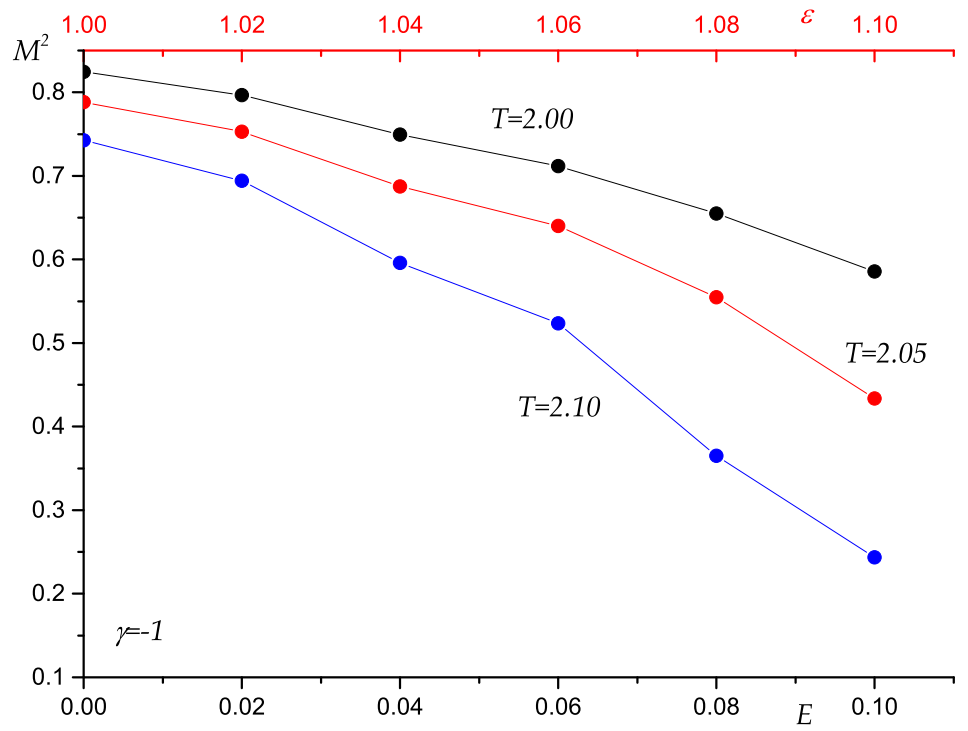

Figure 4. $M^{2}$ versus $E$ at different temperatures $(A=1.0)$.

To consider the change in the phase transition temperature in a thin film under the influence of substrate deformations in an external electric field, let us denote the new temperature of the phase transition $T_{C}$. The magnetization is zero as an order parameter of the system at the phase transition point. Thus, we can write down the following equation:

$$
\alpha\left(T_{C}-T_{0}\right)+D R H E=0 .
$$

The temperature of the phase transition can be recorded as follows:

$$
T_{C}=T_{0}-\frac{D R H}{\alpha} E \text {. }
$$

Thus, an external electric field leads to a Curie temperature decrease in a thin film on a FE substrate. Moreover, the temperature decrease depends linearly on the strength of the electric field. Comparing these results with Figure 1, one can conclude that the mean-field theory is applicable only in weak fields.

\section{Discussion}

We modeled a computer simulation of the FE substrate influence on the condition and magnetic properties of 2D FM nanofilms. Our results show that the uniform deformations of the substrate lead to inhomogeneous deformations of the film. The interaction between the substrate and film atoms causes film deformations. When the substrate is compressed, it forms strip structures in the film. High-density stripes separate stripes with a low concentration of atoms. Substrate stretching leads to the formation of superstructures with an increased concentration of atoms. Heating or an external electric field can cause substrate deformations. In this case, a substrate-induced structural phase transition takes place.

A change in the film structure leads to changes in its magnetic properties. The Curie temperature is shifting, due to the substrate deformations. It decreases with both substrate compression and stretching. The phase transition temperature changing is caused by the dependence of the exchange integral on the distance between the spins. A decrease in the Curie temperature at any deformation of the substrate is explained by inhomogeneous changes in the atoms arrangement in the film. In both tension and compression, there is a competition of two factors. Areas of increased atomic concentration tend to increase the Curie temperature, while areas of decreased atomic concentration lower it. These trends are associated with the linear dependence of the phase transition temperature on the exchange integral. The regions with low atom concentration are dominant, due to the exponential 
decay of exchange integral with distance. Long-range effects can significantly change the dependence of Curie temperature on substrate deformations.

The trends in the magnetic characteristics behavior obtained in the simulation agree with the experimental data.

Author Contributions: Conceptualization, V.S.; funding acquisition, I.B. and V.S.; investigation, S.B.; methodology, I.B. and S.B.; project administration, I.B.; software, I.M.; writing—original draft, S.B. and I.M. All authors have read and agreed to the published version of the manuscript.

Funding: This study was funded by Russian Foundation for Basic Researches, project number 2007-00053, 20-37-70038 and the Ministry of Science and Higher Education of the Russian Federation within the framework of the Russian State Assignment under contract No. 075-00992-21-00. Monte Carlo simulations were performed with the support of the Russian Science Foundation, project number 20-19-00745.

Institutional Review Board Statement: Not applicable.

Informed Consent Statement: Not applicable.

Data Availability Statement: The data presented in this study are available on request from the corresponding author.

Conflicts of Interest: The authors declare no conflict of interest.

\section{References}

1. Sun, Q.; Mahfouzi, F.; Velev, J.P.; Tsymbal, E.Y.; Kioussis, N. Ferroelectric-driven tunable magnetism in ultrathin platinum films. Phys. Rev. Mater. 2020, 4, 124401. [CrossRef]

2. Ghidini, M.; Dhesi, S.S.; Mathur, N.D. Nanoscale magnetoelectric effects revealed by imaging. J. Magn. Magn. Mater. 2021, 520, 167016. [CrossRef]

3. Chen, A.; Huang, H.; Wen, Y.; Liu, W.; Zhang, S.; Kosel, J.; Sun, W.; Zhao, Y.; Lu, Y.; Zhang, X.-X. Giant magnetoelectric effect in perpendicularly magnetized $\mathrm{Pt} / \mathrm{Co} /$ Ta ultrathin films on a ferroelectric substrate. Mater. Horiz. 2020, 7, 2328-2335. [CrossRef]

4. Meenachisundaram, S.; Wakiya, N.; Muthamizhchelvan, C.; Gangopadhyay, P.; Sakamoto, N.; Ponnusamy, S. Enhanced Magnetoelectric Effects in Self-Assembled Hemispherical Close-Packed $\mathrm{CoFe}_{2} \mathrm{O}_{3}-\mathrm{Pb}\left(\mathrm{Zr}_{0.52} \mathrm{Ti}_{0.48}\right) \mathrm{O}_{3}$ Thin Film. J. Electron. Mater. 2021, 50, 1699-1706. [CrossRef]

5. Du, W.; Liu, M.; Su, H.; Zhang, H.; Liu, B.; Meng, H.; Xu, G.; Peng, R.; Tang, X. Wide range voltage-impulse-controlled nonvolatile magnetic memory in magnetoelectric heterostructure. Appl. Phys. Lett. 2020, 117, 222401. [CrossRef]

6. Wang, A.-P.; Song, G.; Zhou, F.-P.; Zhao, L.-N.; Jin, M.; Liu, M.; Zhang, Y.; Hu, L.-L.; Qi, J.; Xu, H.; et al. Strengthened magnetoelectric coupling in $\mathrm{Pb}\left(\mathrm{Zr}_{0.52} \mathrm{Ti}_{0.48}\right) \mathrm{O}_{3} / \mathrm{Ni}$ composite through interface modification with $\mathrm{LaNiO}_{3}$ buffer layer. J. Mater. Sci. Mater. Electron. 2021, 32, 5920-5927. [CrossRef]

7. Shi, M.; Xu, Y.; Zhang, Q.; Yu, Q.; Gu, C.; Zhao, Z.; Guo, L. Impact of heat-treatment conditions on ferroelectric, ferromagnetic and magnetoelectric properties of multi-layered composite films of $\mathrm{Ba}_{0.9} \mathrm{Ca}_{0.1} \mathrm{TiO}_{3} / \mathrm{CoFe}_{2} \mathrm{O}_{4}$. J. Mater. Sci. Mater. Electron. 2019, 30, 19343-19352. [CrossRef]

8. Ivanov, M.S.; Buryakov, A.M.; Vilarinho, P.M.; Mishina, E.D. Impact of compressive and tensile epitaxial strain on transport and nonlinear optical properties of magnetoelectric $\mathrm{BaTiO}_{3}-(\mathrm{LaCa}) \mathrm{MnO}_{3}$ tunnel junction. J. Phys. D Appl. Phys. 2021, 54, 275302. [CrossRef]

9. Apostolova, I.N.; Apostolov, A.T.; Wesselinowa, J.M. Room temperature ferromagnetism in multiferroic $\mathrm{BaCoF}_{4}$ thin films due to surface, substrate and ion doping effects. J. Thin Solid Film. 2021, 722, 138567. [CrossRef]

10. Tongue Magne, G.E.; Keumo Tsiaze, R.M.; Fotué, A.J.; Hounkonnou, N.M.; Fai, L.C. Cumulative effects of fluctuations and magnetoelectric coupling in two-dimensional $\mathrm{RMnO}_{3}(\mathrm{R}=\mathrm{Tb}$, Lu and $\mathrm{Y})$ multiferroics. Phys. Lett. A 2021, 400, 127305. [CrossRef]

11. Frenkel, Y.; Kontorova, T. On the theory of plastic deformation and twinning. Acad. Sci. USSR J. Phys. 1939, 1, 137-149.

12. Nicolenco, A.; Gómez, A.; Chen, X.-Z.; Menéndez, E.; Fornell, J.; Pané, S.; Pellicer, E.; Sort, J. Strain gradient mediated magnetoelectricity in Fe-Ga/P(VDF-TrFE) multiferroic bilayers integrated on silicon. App. Mater. Today 2020, 19, 100579. [CrossRef]

13. Binder, K. Critical Properties from Monte-Carlo Coarse-Graining and Renormalization. Phys. Rev. Lett. 1981, 47, 693. [CrossRef]

14. Wolff, U. Collective Monte Carlo Updating for Spin Systems. Phys. Rev. Lett. 1989, 62, 361. [CrossRef]

15. Landau, D.P.; Binder, K. Phase Diagrams and Multicritical Behavior of a Three-Dimensional Anisotropic Heisenberg Antiferromagnet. Phys. Rev. B 1978, 17, 2328. [CrossRef]

16. Landau, L. On the Theory of Phase Transitions. Phys. Z. Sowjet. 1937, 11, 26. [CrossRef] 